\title{
Ruminal and postruminal digestion of dietary protein and starch in steers: 1. Effects of protein concentration, degradation of protein and energy content of the diet
}

\author{
H.H. Meissner* \\ Animal Nutrition and Animal Products Institute, Private Bag X2, Irene, 1675 Republic of South Africa \\ H.P.F. du Preez \\ Department of Animal and Wildlife Sciences, University of Pretoria, Pretoria, 0002 Republic of South Africa \\ Received 21 August 1995; accepted 26 February 1997
}

\begin{abstract}
Different protein-energy ratios in feedlot diets may effect several combinations of amounts of amino acids and glucose available for absorption in the small intestine. In this regard, the effect of protein concentration and degradation, and energy content of the diet were studied in two $4 \times 4$ Latin Square trials with ruminal and duodenally fistulated steers. In the first trial, steers were fed diets containing $10.0 \mathrm{MJ}$ ME/kg DM at two protein concentrations (105 and $125 \mathrm{~g} \mathrm{CP} / \mathrm{kg} \mathrm{DM})$, each at two rumen degradation levels (60 and $70 \% \mathrm{RDP})$. In the second, the dietary energy coritent was $12.5 \mathrm{MJ} \mathrm{ME} / \mathrm{kg} \mathrm{DM}$, the two protein concentrations were 135 and $155 \mathrm{~g} \mathrm{CP} / \mathrm{kg} \mathrm{DM}$ and the two RDP levels were also 60 and $70 \%$. The protein-energy ratio was the same for both energy levels. All

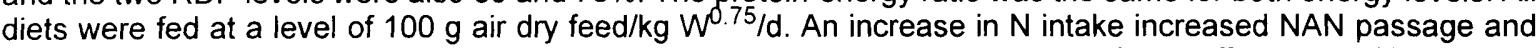
apparent absorption in the lower digestive tract. RDP level did not have a significant effect on NAN passage. The percentage OM that apparently fermented in the rumen $\left(O M D_{R}\right)$ was negatively associated with NAN passage. $O M D_{R}$ was lower on the higher energy diet; the difference being mainly due to the difference in starch digestion. Proportionally less starch was digested in the rumen and more in the lower digestive tract with the higher energy diet, i.e. the higher starch diet. Results indicate that ratios of amino acids and glucose available for absorption from the small intestine vary independently of the dietary protein-energy ratio.
\end{abstract}

Voerkraaldiëte met verskillende proteïen-energieverhoudings kan lei tot verskillende kombinasies van aminosure en glukose in die dunderm. Omstandighede wat tot gunstige verhoudings lei behoort ondersoek te word, en gevolglik is in hierdie studie proteïeninhoud en -degradering tesame met energie-inhoud van die dieet gevarieer. Rumen- en duodenaal gefistuleerde osse is gebruik in ' $n$ eksperimentele ontwerp van twee $4 \times 4$ Latynse Vierkante. In die eerste proef is 'n energie-inhoud van 10.0 MJ ME/kg DM teen twee proteienkonsentrasies (105 en $125 \mathrm{~g} \mathrm{RP} / \mathrm{kg} \mathrm{DM}$ ), elk teen twee rumendegradeerbaarheidspeile (60 en 70\% RDP) gevoer. In die tweede proef is die energie-inhoud verhoog na $12.5 \mathrm{MJ}$ ME/kg DM, die proteïenkonsentrasie na 135 en $155 \mathrm{~g} \mathrm{RP} / \mathrm{kg}$ DM, terwyl die RDP-waarde gehou is op 60 en $70 \%$. Dit het beteken dat die proteien-energieverhouding in die twee proewe dieselfde was. Alle diëte is gevoer teen ' $n$ peil van $100 \mathrm{~g}$ lugdroë voer $/ \mathrm{kg} \mathrm{W}$. $75 / \mathrm{d}$. ' $n$ Toename in $\mathrm{N}$-inname het NAN-vloei en skynbare absorpsie uit die laer spysverteringskanaal verhoog, maar RDP-peil het nie ' $n$ betekenisvolle invloed op NAN-vloei gehad nie. Die persentasie OM wat skynbaar in die rumen verteer het $\left(O M V_{R}\right)$ was negatief gekorreleerd met NAN-vloei. Dit was ook laer op die hoë energiedieet, hoofsaaklik as gevolg van ' $n$ verskil in styselvertering. Verhoudelik is minder stysel in die rumen en meer in die laer spysverteringskanaal in die geval van die hoë energiedieet, dit is die hoër styseldieet, verteer. Die resultate dui daarop dat die verhouding tussen aminosure en glukose in die dunderm onafhankik van die dieetproteïen-energieverhouding varieer.

Keywords: Protein, degradation, starch, digestion, steers

*To whom correspondence should be addressed

\section{Introduction}

The ratio between protein, energy, and protein degradation is apparently important in feedlot diets. This is because the ratio between protein and energy is believed to be critical for microbial protein production (Owens \& Bergen, 1983; Czerkawski, 1986; Newbold \& Rust, 1990), for digestion in the small intestine (Beever et al., 1987) and for tissues to facilitate utilization of substrates in the correct proportions (Kempton et al., 1978; Storm et al., 1983; MacRae \& Lobley, 1986). 'Correct' ratios entering the rumen do not, however, guarantee optimal absorption ratios in the small intestine, because ruminal output of protein and energy differs with feeding system (Meissner et al., 1992), type of starch (Owens et al., 1986), protein degradation (Meissner \& Du Plessis, 1992), and synchronisation of protein and starch (HereraSaldana et al., 1990). More amino acid $\mathrm{N}$ is expected to enter the duodenum when less dietary protein is degraded in the rumen, but starch entry also may increase when less starch is fermented in the rumen (Meissner \& Du Plessis, 1992). Therefore, because more glucose as an end product of starch digestion will be absorbed, more amino acids can be diverted to protein synthesis rather than gluconeogenesis.

A contrasting scenario regarding amino acid availability in the small intestine is possible. Ruminal turnover and dilution rates decrease with increasing concentrate levels in the diet (Stern \& Hoover, 1979; Owens et al., 1984). In addition, ruminal $\mathrm{pH}$ is not effectively controlled, resulting in lowered 
efficiency of microbial growth (Henning et al., 1993). More rumen undegraded protein to supplement microbial amino acids at the duodenum should then be beneficial. If these negative factors do not occur, microbial protein production on higher rumen degraded dietary protein may result in similar amounts or more amino acids available at the duodenum for absorption. Those amino acids that are not required for protein synthesis will be used in glucose production, resulting in less difference in animal production compared with the previous scenario.

This argument suggests that dietary ratios to affect passage of nutrients to the duodenum to influence glucose supply may not be that important because, depending upon starch availability in the small intestine, glucose as end product of starch digestion may substitute for glucose derived from amino acids. We addressed these issues in a digestion trial with feedlot steers in which we altered the dietary protein level, protein degradation and energy (starch) content, but kept the ratio between protein and energy constant.

\section{Materials and Methods \\ Design}

Two separate $4 \times 4$ Latin Square designs were run simultaneously. In the first, steers were fed diets of 10.0 MJ ME/kg DM at two protein content levels ( 105 and $125 \mathrm{~g} \mathrm{CP} / \mathrm{kg} \mathrm{DM}$ ), each at two rumen degradation levels (60 and $70 \% \mathrm{RDP})$. In the second, the energy content was $12.5 \mathrm{MJ} \mathrm{ME} / \mathrm{kg} \mathrm{DM}$, the two protein content levels were 135 and $155 \mathrm{~g} \mathrm{CP} / \mathrm{kg} \mathrm{DM}$, and the RDP levels were also 60 and $70 \%$ (Table 1). All diets were fed at a level of $100 \mathrm{~g}$ air dry feed per $\mathrm{kg}$ metabolic mass $\left(\mathrm{W}^{0.75}\right)$.

\section{Animals, diets and feeding procedures}

Steers weighed $409 \pm 26.5 \mathrm{~kg}$ at the start of the first period and $483 \pm 24.0 \mathrm{~kg}$ at the start of the third period. Because they were getting too fat, they then were fed a roughage diet for one month before the fourth period. Otherwise, three weeks were allowed for adaptation between periods. The steers weighed $448 \pm 19.1 \mathrm{~kg}$ at the start of the fourth period.

Diets consisted primarily of maize byproducts with cottonseed hulls and lucerne pellets as the main fibre source. All feedstuffs (Table 2) were analysed by proximate analysis and their ME contents calculated from equations based on proxi-

Table 1 Design of experiment in terms of energy (ME), crude protein (CP) and rumen degradability of protein (RDP) in the diet DM

\begin{tabular}{cccccc}
\hline & $\begin{array}{c}\text { Diet } \\
\text { Design }\end{array}$ & $\begin{array}{c}\text { Energy } \\
\text { No }\end{array}$ & $\begin{array}{c}\text { Crude protein } \\
\text { ME/kg DM }\end{array}$ & $\begin{array}{c}\text { CP:ME } \\
\text { g CP/kg DM }\end{array}$ & $\begin{array}{c}\text { RDP } \\
\text { g CP/MJ ME }\end{array}$ \\
\hline Latin & 1 & 10.0 & 105 & 10.5 & 60 \\
Square & 2 & 10.0 & 105 & 10.5 & 70 \\
A & 3 & 10.0 & 125 & 12.5 & 60 \\
& 4 & 10.0 & 125 & 12.5 & 70 \\
\hline Latin & 5 & 12.5 & 135 & 10.8 & 60 \\
Square & 6 & 12.5 & 135 & 10.8 & 70 \\
B & 7 & 12.5 & 155 & 12.4 & 60 \\
& 8 & 12.5 & 155 & 12.4 & 60 \\
\hline
\end{tabular}

Table 2 Chemical composition of feedstuffs on a DM basis (\% except ME)

\begin{tabular}{|c|c|c|c|c|c|c|c|c|c|}
\hline Feedstuff & $\mathrm{DM}$ & $\mathrm{N}$ & $\begin{array}{c}\text { RDP } \\
(\mathrm{PO} .05)^{\mathrm{a}}\end{array}$ & $\begin{array}{l}\text { Crude } \\
\text { fibre }\end{array}$ & $\begin{array}{l}\text { Ether } \\
\text { extract }\end{array}$ & Ash & NFE & $\mathrm{TDN}^{\mathrm{b}}$ & $\begin{array}{c}\mathrm{ME}^{\mathrm{b}} \\
(\mathrm{MJ} / \mathrm{kg})\end{array}$ \\
\hline $\begin{array}{l}\text { Cottonseed } \\
\text { hulls }\end{array}$ & 89 & 0.7 & 44 & 60 & 2.0 & 3.7 & 30 & 35 & 5.1 \\
\hline Hominy chop & 91 & 2.2 & 74 & 9.1 & 8.8 & 4.1 & 65 & 85 & 12.9 \\
\hline $\begin{array}{l}\text { Lucerne, } \\
\text { pelleted }\end{array}$ & 90 & 2.1 & 67 & 34 & 1.9 & 12 & 39 & 56 & 8.5 \\
\hline $\begin{array}{l}\text { Maize bran, } \\
\text { high fibre }\end{array}$ & 91 & 1.4 & 71 & 25 & 6.6 & 3.1 & 56 & 73 & 11.0 \\
\hline Maize germ & 91 & 2.2 & 73 & 13 & 15 & 4.5 & 55 & 87 & 13.1 \\
\hline $\begin{array}{l}\text { Maize germ, } \\
\text { defatted }\end{array}$ & 92 & 2.1 & 53 & 10 & 3.2 & 4.1 & 70 & 80 & 12.0 \\
\hline $\begin{array}{l}\text { Maize gluten, } \\
20 \% \text { CP }\end{array}$ & 88 & 3.9 & 89 & 13 & 2.2 & 8.3 & 52 & 71 & 10.7 \\
\hline $\begin{array}{l}\text { Maize gluten, } \\
60 \% \text { CP }\end{array}$ & 93 & 11 & 32 & 2.2 & 1.1 & 2.6 & 25 & 76 & 11.4 \\
\hline Maize meal & 89 & 1.8 & 75 & 4.7 & 4.0 & 1.4 & 79 & 86 & 13.0 \\
\hline Sorghum meal & 89 & 1.6 & 69 & 4.8 & 3.7 & 1.7 & 80 & 87 & 13.2 \\
\hline Wheat bran & 89 & 2.8 & 92 & 15 & 5.5 & 6.6 & 56 & 73 & 11.1 \\
\hline
\end{tabular}

${ }^{a}$ Degradation at a fractional outflow rate of $0.05 / \mathrm{h}$

${ }^{\mathrm{b}}$ Calculated from Kearl (1982)

mate analysis constituents (Kearl, 1982). Starch was determined only on the mixed diets. Degradation of protein was determined by nylon bag technique as described by Meissner et al. (1992). Effective degradation was calculated at a fractional outflow rate of $0.05 / \mathrm{h}$. Diet compositions are shown in Table 3.

The eight steers were housed in metabolism stalls which facilitated faeces and urine collection. They were fitted with a $100 \mathrm{~mm}$ diameter rumen cannula and a simple cannula in the proximal duodenum. Feeding was at 06:00, 12:00, 18:00 and 00:00 to approach steady state conditions, and they were fed after rumen and duodenal sampling. Orts were accumulated for the week of measurement. These amounted to less than $5 \%$ of feed allocated. The composition of the orts did not differ appreciably from that of the diets.

\section{Measurements}

Rumen digesta content was determined by emptying the rumen at specific times (Pienaar et al., 1980). Digesta passage was measured by the double marker technique (Faichney, 1980) with $\mathrm{Na}$-dichromate as particulate and Co-EDTA as fluid marker (Coleman et al., 1984). These markers were mixed into the feed after a primer dose had been introduced through the rumen cannula (Meissner \& Du Plessis, 1992). Duodenal digesta samples were collected over four days at randomly allotted times to simulate sampling every $2 \mathrm{~h}$ in one 24-h cycle; samples were pooled for analyses. Faeces were collected in toto.

Passage and apparent digestibility of OM, N, non-ammonia $\mathrm{N}$ (NAN) and starch were, where applicable, determined between mouth and duodenum and between duodenum and faeces.

Additionally, rumen volatile fatty acids (VFA), $\mathrm{pH}$ and 
Table 3 Diet compositions (\%, air dry)

\begin{tabular}{lcccccccc}
\hline & \multicolumn{7}{c}{ Diet 0} \\
\cline { 2 - 8 } Feedstuff & 1 & 2 & 3 & 4 & 5 & 6 & 7 & 8 \\
\hline Cottonseed hulls & 14.00 & 13.00 & 13.00 & 13.00 & - & - & - & - \\
Hominy chop & - & - & 25.64 & 4.86 & 43.12 & - & - & - \\
Lucerne, pelleted & 16.53 & 37.16 & 18.76 & 27.24 & 3.83 & 4.09 & 2.50 & 2.06 \\
Maize bran, high fibre & - & - & - & - & 2.86 & 1.23 & - & 2.05 \\
Maize germ & 1.33 & - & - & 44.31 & - & 45.14 & 23.99 & 45.05 \\
Maize germ, defatted & 38.82 & - & 34.37 & - & - & - & - & - \\
Maize gluten, $20 \% \mathrm{CP}$ & - & - & - & 3.74 & - & - & - & - \\
Maize gluten, $60 \% \mathrm{CP}$ & - & - & 1.75 & 0.70 & - & - & 7.53 & - \\
Maize meal & - & 16.25 & - & - & - & 42.26 & 50.40 & 42.98 \\
Sorghum meal & 22.26 & 22.51 & - & - & 43.09 & - & 8.51 & - \\
Wheat bran & - & 4.37 & - & - & - & - & - & - \\
Molasses & 5.00 & 5.00 & 5.00 & 5.0 & 5.00 & 5.00 & 5.00 & 5.00 \\
Urea & - & - & - & - & - & 0.25 & - & 0.77 \\
Limestone & 1.34 & 0.31 & 0.72 & 0.55 & 1.21 & 1.42 & 1.45 & 1.47 \\
Monocalcium & & & & & & & & \\
phosphate & 0.12 & 0.80 & 0.17 & - & 0.27 & - & - & - \\
Salt & 0.50 & 0.50 & 0.50 & 0.50 & 0.50 & 0.50 & 0.50 & 0.50 \\
Vitamin-mineral & & & & & & & & \\
premix* & 0.10 & 0.10 & 0.10 & 0.10 & 0.10 & 0.10 & 0.10 & 0.10 \\
\hline
\end{tabular}

* Included a standard antibiotic and ionophore

NH3-N concentration were determined from pooled samples. These samples were collected simultaneously with the duodenal samples. Samples for VFA determination were preserved with $\mathrm{NaOH}$ and samples for $\mathrm{NH} 3-\mathrm{N}$ determination with $\mathrm{H} 2 \mathrm{SO} 4$. Ruminal $\mathrm{pH}$ was measured in the supernatant fluid after filtration.

\section{Chemical analyses}

Dry matter contents of feeds, rumen, duodenal and faecal samples were obtained by drying to constant mass at $60^{\circ} \mathrm{C}$, and $\mathrm{OM}$ after incineration at $550^{\circ} \mathrm{C}$ to determine ash. Nitrogen was determined by Kjeldahl and rumen and duodenal NH3-N concentration by auto-analyser (Technicon Auto Analyser II. Indust. Method 334-74A). Feed, duodenal and faecal starch was determined by the a-amylase method (MacRae \& Armstrong, 1968) and rumen VFA by gas chromatography. Non-ammonia $\mathrm{N}$ was calculated as the difference between total $\mathrm{N}$ and $\mathrm{NH} 3-\mathrm{N}$ in duodenal samples.

\section{Statistical analyses}

The Latin Squares were analysed with treatments and periods as factors using the General Linear Models programme (SAS, 1985). The data of both were also combined to examine differences between dietary ME contents and two-way $(\mathrm{ME} \times$ $\mathrm{CP}, \mathrm{ME} \times \mathrm{RDP}, \mathrm{CP} \times \mathrm{RDP})$ and three-way $(\mathrm{ME} \times \mathrm{CP} \times \mathrm{RDP})$ interactions. (See ANOVA, Table 4.) The three-way interaction was never significant and is therefore not included in the results. Tukey's $t$ test was the basis of comparison. The combination analysis has limitations because energy content and animals would have been confounded. The purpose, however, was to examine whether, apart from differences between
Table 4 Skeleton ANOVA of the combined analysis for the effect of energy (ME) content

\begin{tabular}{lc}
\hline Source of variation & $d f$ \\
\hline $\mathrm{CFM}$ & 1 \\
$\mathrm{ME}$ & 1 \\
Error (a) & 0 \\
Steers in Latin squares & 3 \\
Periods (common) & 1 \\
$\mathrm{RDP}$ & 1 \\
$\mathrm{RDP} \times \mathrm{ME}$ & 1 \\
$\mathrm{CP}($ linear $)$ & 1 \\
$\mathrm{CP}($ linear $) \times$ ME & 1 \\
$\mathrm{RDP} \times \mathrm{CP}$ (linear) & 1 \\
$\mathrm{RDP} \times \mathrm{CP}($ linear $) \times \mathrm{ME}$ & $(6+6)+3=15$ \\
Error $(\mathrm{b})$ & $2 \times 16=32$ \\
Total $($ uncorrected) & \\
\hline
\end{tabular}

energy contents, the proportional responses to $\mathrm{CP}$ and $\mathrm{RDP}$ were similar.

\section{Results}

Table 5 shows the results for the $10.0 \mathrm{MJ}$ ME $/ \mathrm{kg}$ DM (low energy) diet, Table 6 the results for the $12.5 \mathrm{MJ} \mathrm{ME} / \mathrm{kg} \mathrm{DM}$ (high energy) diet and Table 7 the results of the combined data to compare energy contents and interaction with protein and RDP levels.

Organic matter intake, as intended, did not differ significantly between treatments. Starch intake was not significantly different between $\mathrm{CP}$ and RDP levels for the low energy diet (Table 5) but it was higher on the lower $\mathrm{CP}(p \leq 0.006)$ and $\operatorname{RDP}(p \leq 0.04)$ diets of the high energy diet (Table 6). This was a function of the specific combinations of feedstuffs used to formulate the diets. More starch was consumed with the high energy diet $(p \leq 0.01)$ as expected and the ME $\times$ RDP interaction also was significant (Table 7). Nitrogen intake, as intended, differed between the low and high protein levels, respectively at $p \leq 0.004$ and $p \leq 0.0003$ for the low and high energy diets, but $\mathrm{N}$ intake did not differ significantly between RDP levels, nor was the interaction with ME content significant.

As far as total tract digestibility is concerned, the apparent digestibility of OM did not differ significantly between $\mathrm{CP}$ or RDP levels and, surprisingly, not between dietary energy contents (Table 7). Starch digestibility was higher $(p \leq 0.01)$ on the lower CP level of the high energy diet (Table 6), but was not significantly influenced by RDP level or energy content. None of the interactions was significant. The apparent $\mathrm{N}$ digestibility usually reflects the amount of $\mathrm{N}$ in the diet, being higher with higher $\mathrm{N}$ content. The difference was significant $(p \leq 0.012)$ for the protein content of the high energy diet (Table 6) and the protein content in the low $v$ s the high energy diets (Table 7).

Rumen $\mathrm{pH}$ was higher $(p \leq 0.0002)$ on the $70 \% \mathrm{RDP}$ diets of the low energy diet (Table 5) and tended to be higher ( $p \leq$ 0.07 ) on the high energy diet (Table 6). Between energy contents, rumen $\mathrm{pH}$ was lower on the high energy diet $(p \leq 0.002)$ (Table 7). Rumen NH3-N levels were also affected by RDP 
Table 5 The effect of level of protein and degradability on ruminal and postruminal digestion in steers receiving diets of $10.0 \mathrm{MJ} \mathrm{ME} / \mathrm{kg} \mathrm{DM}$

\begin{tabular}{|c|c|c|c|c|c|c|c|}
\hline \multirow[b]{3}{*}{ Item $(n=16)$} & \multirow{2}{*}{\multicolumn{2}{|c|}{$\mathrm{CP}, \mathrm{g} / \mathrm{kg} \mathrm{DM}$}} & \multirow{2}{*}{\multicolumn{2}{|c|}{ RDP, $\%$}} & \multirow[b]{3}{*}{$S D$} & \multicolumn{2}{|c|}{$\mathrm{PR} \geq \mathrm{F}$} \\
\hline & & & & & & \multirow{2}{*}{\multicolumn{2}{|c|}{$\begin{array}{l}\text { Protein } \\
\text { content Degrad }\end{array}$}} \\
\hline & 105 & 125 & 60 & 70 & & & \\
\hline \multicolumn{8}{|l|}{ Intake } \\
\hline $\mathrm{OM}, \mathrm{kg} / \mathrm{d}$ & 8.12 & 8.07 & 8.02 & 8.17 & 0.24 & 0.84 & 0.57 \\
\hline Starch, kg/d & 1.84 & 1.76 & 1.74 & 1.86 & 0.15 & 0.59 & 0.44 \\
\hline $\mathrm{N}, \mathrm{g} / \mathrm{d}$ & $154^{\mathrm{a}}$ & $174^{\mathrm{b}}$ & 159 & 169 & 4.90 & 0.004 & 0.42 \\
\hline \multicolumn{8}{|l|}{ Digestibility } \\
\hline OM, \% & 79.0 & 77.7 & 77.9 & 78.8 & 1.94 & 0.52 & 0.64 \\
\hline Starch, $\%$ & 93.7 & 93.3 & 93.1 & 93.9 & 1.39 & 0.76 & 0.58 \\
\hline $\mathrm{N}, \%$ & 74.0 & 74.3 & 72.8 & 75.5 & 1.47 & 0.87 & 0.10 \\
\hline \multicolumn{8}{|l|}{ Rumen } \\
\hline $\mathrm{pH}$ & 6.42 & 6.39 & $6.35^{\mathrm{c}}$ & $6.46^{\mathrm{d}}$ & 0.10 & 0.39 & 0.0002 \\
\hline $\mathrm{NH} 3-\mathrm{N}, \mathrm{mmol} / \mathrm{l}$ & $6.28^{\mathrm{a}}$ & $7.89^{\mathrm{b}}$ & $6.41^{\mathrm{c}}$ & $7.76^{\mathrm{d}}$ & 0.94 & 0.001 & 0.006 \\
\hline VFA, mmol/l & 24.2 & 24.4 & 24.9 & 23.7 & 1.70 & 0.88 & 0.15 \\
\hline $\mathrm{C}_{2}, \mathrm{mmol} / \mathrm{l}$ & 16.1 & 16.1 & 16.4 & 15.7 & 1.23 & 0.97 & 0.30 \\
\hline $\mathrm{C}_{3}, \mathrm{mmol} / \mathrm{l}$ & 3.75 & 3.98 & 3.97 & 3.75 & 0.56 & 0.42 & 0.43 \\
\hline $\mathrm{C}_{4}, \mathrm{rnmol} / \mathrm{l}$ & 3.82 & 3.62 & 3.88 & 3.55 & 0.37 & 0.28 & 0.09 \\
\hline $\mathrm{C}_{5}, \mathrm{mmol} / \mathrm{l}$ & $0.62^{\mathrm{a}}$ & $0.70^{\mathrm{b}}$ & 0.68 & 0.64 & 0.07 & 0.024 & 0.32 \\
\hline $\mathrm{C}_{2}: \mathrm{C}_{3}$ & 4.49 & 4.38 & 4.35 & 4.52 & 0.50 & 0.50 & 0.65 \\
\hline $\mathrm{C}_{2}: \mathrm{C}_{4}$ & $4.25^{\mathrm{a}}$ & $4.57^{b}$ & 4.27 & 4.55 & 0.29 & 0.029 & 0.06 \\
\hline OM digest., $\mathrm{kg} / \mathrm{d}$ & 5.52 & 5.27 & 5.26 & 5.53 & 0.36 & 0.50 & 0.48 \\
\hline OM digest., $\%$ of & 68.0 & 65.3 & 65.6 & 67.7 & 2.89 & 0.35 & 0.44 \\
\hline Starch digest., $\mathrm{kg} / \mathrm{d}$ & 1.5 & 1.54 & 1.53 & 1.59 & 0.15 & 0.74 & 0.66 \\
\hline Starch digest.. \% of intake & 86.4 & 87.5 & 87.9 & 85.5 & 2.83 & 0.80 & 0.53 \\
\hline $\mathrm{N}$ digest., $\mathrm{g} / \mathrm{d}$ & 35.6 & 35.4 & 35.0 & 36.0 & 10.8 & 0.99 & 0.93 \\
\hline $\mathrm{N}$ digest. $\%$ of intake & 23.1 & 20.3 & 22.0 & 21.3 & 5.86 & 0.63 & 0.88 \\
\hline \multicolumn{8}{|l|}{ Passage to duodenum } \\
\hline Digesta, $\mathrm{kg} / \mathrm{d}$ & 103 & 107 & 103 & 107 & 10.7 & 0.69 & 0.68 \\
\hline $\mathrm{OM}, \mathrm{kg} / \mathrm{d}$ & 2.60 & 2.80 & 2.77 & 2.64 & 0.19 & 0.30 & 0.50 \\
\hline Starch, kg/d & 0.25 & 0.22 & 0.21 & 0.27 & 0.11 & 0.63 & 0.37 \\
\hline $\mathrm{NH} 3-\mathrm{N}, \mathrm{g} / \mathrm{d}$ & $3.52^{\mathrm{a}}$ & $5.10^{\mathrm{b}}$ & 4.41 & 4.21 & 0.64 & 0.032 & 0.76 \\
\hline $\mathrm{NAN}, \mathrm{g} / \mathrm{d}$ & $116^{\mathrm{a}}$ & $133^{\mathrm{b}}$ & 123 & 126 & 7.26 & 0.043 & 0.66 \\
\hline \multicolumn{8}{|l|}{ Postruminal digestion } \\
\hline $\mathrm{OM}, \mathrm{kg} / \mathrm{d}$ & 0.92 & 1.02 & 1.01 & 0.92 & 0.23 & 0.66 & 0.71 \\
\hline OM, \% of intake & 11.3 & 12.6 & 12.6 & 11.3 & 3.03 & 0.62 & 0.66 \\
\hline Starch, $\mathrm{kg} / \mathrm{d}$ & 0.14 & 0.10 & 0.09 & 0.15 & 0.04 & 0.41 & 0.17 \\
\hline Starch, \% of intake & 7.61 & 5.68 & 5.17 & 8.06 & 2.27 & 0.61 & 0.27 \\
\hline NAN, g/d & 76.8 & 89.5 & 79.9 & 86.3 & 8.03 & 0.13 & 0.45 \\
\hline NAN, $\%$ of $N$ intake & 49.9 & 51.4 & 50.3 & 51.1 & 5.91 & 0.61 & 0.77 \\
\hline
\end{tabular}

Values with different superscripts between CP contents (a,b) and RDP levels (c,d) differ significantly

level and energy content. They were higher on the $70 \%$ RDP diets with both the low $(p \leq 0.006)$ and the high energy diets $(p \leq 0.0001)$. They also were higher with the higher protein content with the low energy diets $(p \leq 0.001)$ but not the high energy diet, reflecting a significant $\mathrm{ME} \times \mathrm{CP}$ interaction (Table 7). Total volatile fatty acid concentration in the rumen was not significantly affected by either protein content, RDP
Table 6 The effect of level of protein and degradability on ruminal and postruminal digestion in steers receiving diets of $12.5 \mathrm{MJ} \mathrm{ME} / \mathrm{kg} \mathrm{DM}$

\begin{tabular}{|c|c|c|c|c|c|c|c|}
\hline \multirow[b]{3}{*}{ Item $(n=16)$} & \multirow{2}{*}{\multicolumn{2}{|c|}{$\mathrm{CP}, \mathrm{g} / \mathrm{kg} \mathrm{DM}$}} & \multirow{2}{*}{\multicolumn{2}{|c|}{ RDP, $\%$}} & \multirow[b]{3}{*}{$S D$} & \multicolumn{2}{|c|}{$\mathrm{PR} \geq \mathrm{F}$} \\
\hline & & & & & & \multirow{2}{*}{\multicolumn{2}{|c|}{$\begin{array}{l}\text { Protein } \\
\text { content Degrad }\end{array}$}} \\
\hline & 135 & 155 & 60 & 70 & & & \\
\hline \multicolumn{8}{|l|}{ Intake } \\
\hline $\mathrm{OM}, \mathrm{kg} / \mathrm{d}$ & 7.98 & 8.06 & 7.95 & 8.09 & 0.23 & 0.75 & 0.55 \\
\hline Starch, $\mathrm{kg} / \mathrm{d}$ & $2.37^{\mathrm{b}}$ & $1.86^{\mathrm{a}}$ & $2.29^{\mathrm{d}}$ & $1.94^{\mathrm{c}}$ & 0.15 & 0.006 & 0.04 \\
\hline $\mathrm{N}, \mathrm{g} / \mathrm{d}$ & $185^{\mathrm{a}}$ & $226^{\mathrm{b}}$ & 202 & 209 & 6.80 & 0.0003 & 0.50 \\
\hline \multicolumn{8}{|l|}{ Digestibility } \\
\hline OM, \% & 77.6 & 78.5 & 77.7 & 78.4 & 1.49 & 0.53 & 0.64 \\
\hline Starch, \% & $95.9^{\mathrm{b}}$ & $93.1^{\mathrm{a}}$ & 94.3 & 94.6 & 0.97 & 0.016 & 0.78 \\
\hline $\mathrm{N}, \%$ & $76.1^{\mathrm{a}}$ & $81.6^{\mathrm{b}}$ & 78.6 & 79.1 & 1.82 & 0.012 & 0.78 \\
\hline \multicolumn{8}{|l|}{ Rumen } \\
\hline $\mathrm{pH}$ & 6.21 & 6.26 & 6.20 & 6.26 & 0.13 & 0.187 & 0.07 \\
\hline $\mathrm{NH}_{3}-\mathrm{N}, \mathrm{mmol} / \mathrm{l}$ & 8.57 & 8.17 & $7.08^{\mathrm{c}}$ & $9.66^{d}$ & 1.25 & 0.53 & 0.0001 \\
\hline VFA, mmol/l & 23.4 & 22.9 & 23.5 & 22.8 & 1.87 & 0.62 & 0.43 \\
\hline $\mathrm{C}_{2}, \mathrm{mmol} / \mathrm{l}$ & 14.8 & 14.6 & 14.8 & 14.6 & 1.25 & 0.69 & 0.66 \\
\hline $\mathrm{C}_{3}, \mathrm{mmol} / \mathrm{l}$ & 4.60 & 4.84 & 4.97 & 4.47 & 0.60 & 0.43 & 0.11 \\
\hline $\mathrm{C}_{4}, \mathrm{mmol} / \mathrm{l}$ & $3.20^{\mathrm{b}}$ & $2.65^{\mathrm{a}}$ & 2.89 & 2.95 & 0.38 & 0.006 & 0.74 \\
\hline $\mathrm{C}_{5}, \mathrm{mmol} / \mathrm{l}$ & 0.76 & 0.85 & 0.82 & 0.19 & 0.09 & 0.06 & 0.53 \\
\hline $\mathrm{C}_{2}: \mathrm{C}_{3}$ & 3.36 & 3.32 & 3.31 & 3.37 & 0.42 & 0.87 & 0.79 \\
\hline $\mathrm{C}_{2}: \mathrm{C}_{4}$ & $4.71^{\mathrm{a}}$ & $5.85^{\mathrm{b}}$ & 5.40 & 5.16 & 0.58 & 0.0002 & 0.41 \\
\hline OM digest., $\mathrm{kg} / \mathrm{d}$ & 4.66 & 4.52 & 4.53 & 4.65 & 0.25 & 0.58 & 0.63 \\
\hline OM digest., $\%$ of intake & 58.4 & 56.1 & 57.0 & 57.5 & 2.54 & 0.39 & 0.79 \\
\hline Starch digest., $\mathrm{kg} / \mathrm{d}$ & $1.95^{\mathrm{b}}$ & $1.47^{\mathrm{a}}$ & 1.80 & 1.62 & 0.18 & 0.022 & 0.33 \\
\hline Starch digest., $\%$ of intake & 82.3 & 79.0 & 78.6 & 83.5 & 4.60 & 0.58 & 0.24 \\
\hline$N$ digest., $g / d$ & 37.6 & 44.8 & 34.3 & 48.1 & 12.2 & 0.57 & 0.29 \\
\hline $\mathrm{N}$ digest., $\%$ of intake & 20.3 & 19.8 & 17.0 & 23.0 & 5.04 & 0.99 & 0.20 \\
\hline \multicolumn{8}{|l|}{ Passage to duodenum } \\
\hline Digesta, $\mathrm{kg} / \mathrm{d}$ & 85.3 & 96.1 & 84.7 & 96.7 & 7.48 & 0.18 & 0.14 \\
\hline $\mathrm{OM}, \mathrm{kg} / \mathrm{d}$ & 3.3 & 3.54 & 3.42 & 3.44 & 0.23 & 0.38 & 0.93 \\
\hline Starch, $\mathrm{kg} / \mathrm{d}$ & 0.42 & 0.39 & 0.49 & 0.32 & 0.12 & 0.26 & 0.16 \\
\hline $\mathrm{NH}_{3}-\mathrm{N}, \mathrm{g} / \mathrm{d}$ & 3.65 & 6.42 & 4.23 & 5.84 & 1.38 & 0.07 & 0.27 \\
\hline NAN, g/d & $150^{\mathrm{a}}$ & $177^{b}$ & 168 & 159 & 8.32 & 0.007 & 0.25 \\
\hline \multicolumn{8}{|l|}{ Postruminal digestion } \\
\hline $\mathrm{OM}, \mathrm{kg} / \mathrm{d}$ & 1.54 & 1.81 & 1.65 & 1.70 & 0.16 & 0.13 & 0.80 \\
\hline OM, $\%$ of intake & 19.3 & 22.5 & 20.8 & 21.0 & 1.92 & 0.12 & 0.98 \\
\hline Starch, $\mathrm{kg} / \mathrm{d}$ & 0.32 & 0.25 & 0.36 & 0.21 & 0.08 & 0.42 & 0.11 \\
\hline Starch, \% of intake & 13.5 & 13.4 & 15.7 & 10.8 & 4.57 & 0.97 & 0.26 \\
\hline NAN, g/d & $103^{\mathrm{a}}$ & $135^{b}$ & 125 & 116 & 10.1 & 0.009 & 0.37 \\
\hline NAN, $\%$ of $N$ intake & 55.7 & 59.7 & 61.9 & 55.5 & 5.22 & 0.32 & 0.26 \\
\hline
\end{tabular}

Values with different superscripts between CP contents (a,b) and RDP levels $(c, d)$ differ significantly

level or energy content. Between individual VFA's and ratios, small differences were detected between CP contents (Table 5 and 6) and major differences between energy contents (Table 7). Rumen digestibility of OM, starch or $\mathrm{N}$ was not significantly affected by CP or RDP level, but significantly more $\mathrm{OM}$ and starch as a proportion of intake (Table 7) were digested in the rumen on the low than the high energy diet $(p$ 
Table 7 The effect of energy and interactions with level of protein and degradability on ruminal and postruminal digestion in steers receiving diets varying in $M E, C P$ and RDP

\begin{tabular}{|c|c|c|c|c|c|}
\hline \multirow[b]{2}{*}{ Item $(n=32)$} & \multicolumn{2}{|c|}{$\mathrm{ME} \mathrm{MJ/kg} \mathrm{DM}$} & \multicolumn{3}{|c|}{ Interaction } \\
\hline & 10.0 & 12.5 & $\mathrm{ME} \times \mathrm{CP}$ & $\mathrm{ME} \times \mathrm{RDP}$ & $\mathrm{CP} \times \mathrm{RDP}$ \\
\hline \multicolumn{6}{|l|}{ Intake } \\
\hline $\mathrm{OM}, \mathrm{kg} / \mathrm{d}$ & 8.09 & 8.02 & NS & NS & NS \\
\hline Starch, $\mathrm{kg} / \mathrm{d}$ & $1.80^{\mathrm{a}}$ & $2.12^{\mathrm{b}}$ & NS & $*$ & NS \\
\hline $\mathrm{N}, \mathrm{g} / \mathrm{d}$ & $164^{\mathrm{a}}$ & $207^{b}$ & NS & NS & NS \\
\hline \multicolumn{6}{|l|}{ Digestibility } \\
\hline $\mathrm{OM}, \%$ & 78.4 & 78.0 & NS & NS & NS \\
\hline Starch, \% & 93.5 & 94.5 & NS & NS & NS \\
\hline $\mathrm{N}, \%$ & $74.2^{\mathrm{a}}$ & $78.8^{\mathrm{b}}$ & NS & NS & NS \\
\hline \multicolumn{6}{|l|}{ Rumen } \\
\hline $\mathrm{pH}$ & $6.41^{b}$ & $6.23^{\mathrm{a}}$ & NS & NS & NS \\
\hline $\mathrm{NH}_{3}-\mathrm{N}, \mathrm{mmol} / \mathrm{l}$ & $7.08^{\mathrm{a}}$ & $8.37^{b}$ & * & NS & NS \\
\hline $\mathrm{VFA}, \mathrm{mmol} / 1$ & 24.3 & 23.3 & NS & NS & NS \\
\hline $\mathrm{C}_{2}, \mathrm{mmol} / \mathrm{l}$ & $16.1^{\mathrm{b}}$ & $14.7^{\mathrm{a}}$ & NS & NS & NS \\
\hline $\mathrm{C}_{3}, \mathrm{mmol} / \mathrm{l}$ & $3.86^{\mathrm{a}}$ & $4.72^{\mathrm{b}}$ & NS & NS & NS \\
\hline $\mathrm{C}_{4}, \mathrm{mmol} / \mathrm{l}$ & $3.71^{\mathrm{b}}$ & $2.92^{\mathrm{a}}$ & NS & NS & NS \\
\hline $\mathrm{C}_{5}, \mathrm{mmol} / \mathrm{l}$ & $0.66^{\mathrm{a}}$ & $0.80^{\mathrm{b}}$ & NS & NS & NS \\
\hline $\mathrm{C}_{2}: \mathrm{C}_{3}$ & $4.43^{b}$ & $3.34^{\mathrm{a}}$ & NS & NS & NS \\
\hline $\mathrm{C}_{2}: \mathrm{C}_{4}$ & $4.41^{\mathrm{a}}$ & $5.28^{\mathrm{b}}$ & NS & NS & NS \\
\hline OM digest., $\mathrm{kg} / \mathrm{d}$ & $5.39^{\mathrm{b}}$ & $4.59^{\mathrm{a}}$ & NS & NS & NS \\
\hline OM digest., $\%$ of intake & $66.6^{\mathrm{b}}$ & $57.2^{\mathrm{a}}$ & NS & NS & NS \\
\hline Starch digest., $\mathrm{kg} / \mathrm{d}$ & 1.56 & 1.71 & NS & NS & NS \\
\hline Starch digest., $\%$ of intake & $86.7^{\mathrm{b}}$ & $80.7^{\mathrm{a}}$ & NS & $*$ & $*$ \\
\hline $\mathrm{N}$ digest., g/d & 35.5 & 41.2 & NS & NS & NS \\
\hline $\mathrm{N}$ digest., $\%$ of intake & 21.6 & 19.9 & NS & NS & NS \\
\hline \multicolumn{6}{|l|}{ Passage to duodenum } \\
\hline Digesta, $\mathrm{kg} / \mathrm{d}$ & $105^{\mathrm{b}}$ & $90.7^{\mathrm{a}}$ & NS & NS & NS \\
\hline $\mathrm{OM}, \mathrm{kg} / \mathrm{d}$ & $2.70^{\mathrm{a}}$ & $3.43^{b}$ & NS & NS & NS \\
\hline Starch, $\mathrm{kg} / \mathrm{d}$ & $0.24^{\mathrm{a}}$ & $0.41^{b}$ & NS & * & NS \\
\hline $\mathrm{NH}_{3}-\mathrm{N}, \mathrm{g} / \mathrm{d}$ & 4.31 & 5.04 & NS & * & NS \\
\hline NAN, g/d & $125^{\mathrm{a}}$ & $164^{b}$ & NS & NS & NS \\
\hline \multicolumn{6}{|l|}{ Postruminal digestion } \\
\hline $\mathrm{OM}, \mathrm{kg} / \mathrm{d}$ & $0.96^{\mathrm{a}}$ & $1.68^{\mathrm{b}}$ & NS & NS & NS \\
\hline $\mathrm{OM}, \%$ of intake & $11.9^{\mathrm{a}}$ & $20.9^{\mathrm{b}}$ & NS & NS & NS \\
\hline Starch, $\mathrm{kg} / \mathrm{d}$ & $0.12^{\mathrm{a}}$ & $0.29^{b}$ & NS & * & NS \\
\hline Starch, $\%$ of intake & $6.67^{\mathrm{a}}$ & $13.7^{\mathrm{b}}$ & NS & * & NS \\
\hline $\mathrm{NAN}, \mathrm{g} / \mathrm{d}$ & $83.1^{\mathrm{a}}$ & $119^{b}$ & NS & NS & NS \\
\hline NAN, $\%$ of $N$ intake & 50.7 & 57.5 & NS & NS & NS \\
\hline
\end{tabular}

a.b Values with different superscripts differ significantly

NS - interaction not significant

* - interaction significant

$\leq 0.0001$ and $p \leq 0.037$ respectively). This probably reflects differences in rumen retention time between the two energy contents.

Passage of total wet digesta, OM and starch to the duodenum was not significantly influenced by $\mathrm{CP}$ or RDP level but it was significantly affected by energy content of the diet
(Table 7). Less digesta passed to the duodenum on the high energy diet $(p \leq 0.03)$ but more OM $(p \leq 0.0001)$ and starch $(p \leq 0.002)$. The ME $\times$ RDP interaction was also significant for starch passage, indicating that more starch passed to the duodenum with the $60 \%$ RDP for the high energy diet but with the $70 \%$ RDP for the low energy diet. Both $\mathrm{NH}_{3}-\mathrm{N}$ and NAN passage to the duodenum were higher on the higher $\mathrm{CP}$ diets (Tables 5 and 6 ) and NAN passage was also higher on the high energy diet $(p \leq 0.0001)$ (Table 7). These parameters were, however, not significantly influenced by RDP levels (Tables 5 and 6).

Postruminal digestion of OM, starch and NAN as a percentage of $\mathrm{N}$ intake was not significantly influenced by $\mathrm{CP}$ or RDP level, but energy content of the diet had a major effect (Table 7). More OM $(p \leq 0.0001)$, starch $(p \leq 0.002)$ and NAN $(p \leq 0.0001)$ were digested on the high energy diet, although the difference for NAN digestion was reduced ( $p \leq$ 0.100 ) if corrected for the difference in $N$ intake between the high and low energy diets. The ME $\times$ RDP interaction for postruminal starch digestion was also significant, indicating that more starch was digested with the $60 \%$ RDP for the high energy diet but with the $70 \%$ RDP for the low energy diet.

\section{Discussion}

The difference between the two dietary energy concentrations was less than anticipated (Table 7). Total OM digestibility did not differ significantly; in fact, it was similar. A contributing factor could have been that ME contents of the diets were calculated from the proximate analyses of their constituents according to formulae of Kearl (1982) (Table 2). Both the production and apparent absorption of end products do, however, suggest significant differences at the ME and particularly at the NE level. The concentration and thereby, presumably, the production of propionic acid, which should result in less $\mathrm{CH}_{4}$ energy loss, was greater with the high energy diets. Postruminally, more NAN and starch were digested with the high energy diets. Therefore, quantitatively, more glucose precursors should have been available at the tissue level on the high energy diets.

Total starch digestibility was not affected by energy content of the diets (Table 7), but it was higher with the lower protein concentration of the high energy diets (Table 6). This could have resulted because, owing to feedstuff composition, more starch coincidentally was consumed and therefore digested in the rumen on the lower protein diet. If more starch is digested in the rumen, total starch digestibility usually increases (Streeter et al., 1991). In general, total starch digestibility was about $94 \%$, which corresponds with the $94.2 \%$ reported by Meissner \& Du Plessis (1992) and the 94-95\% for high-lysine and normal-lysine cultivars found by Leeuw $\&$ Coetzer (unpublished). Thus, $94 \%$ appears to be fairly typical of starch digestibility of maize-based feedlot diets in South Africa. It is, however, generally lower than the $99 \%$ reported for total tract starch digestibilities of flaked and high moisture maize in the USA (Muntifering et al., 1981; Spicer et al., 1986; Streeter \& Mathis, 1995). Corresponding digestibilities for whole maize may be as low as $92 \%$.

Proportionally less starch and OM were digested in the rumen and proportionally more in the lower digestive tract on the high energy diets. Thus, with more starch in the present 
diets, more passed to the duodenum. The passage of starch to the duodenum may also be influenced by RDP level. Meissner \& Du Plessis (1992) found that more starch passed to the duodenum on a $62 \%$ RDP diet when fish meal was the supplement compared to a 74\% RDP diet when urea was the supplement. This may be due to less extensive and/or slower fermentation on the fish meal diet. Streeter \& Mathis (1995) also reported that starch passage to the duodenum increased with fish meal supplementation but the response was quadratic, indicating that the level is important and that responses do not always occur or may be negative. In the present study, starch passage to the duodenum was not affected by RDP level on both dietary energy concentrations. Where increased passage did occur, it coincided with proportionally less OM digested in the rumen, which is a function of rumen retention time rather than RDP level, but which may, according to the results above, be modified by RDP level.

Passage of NAN to the duodenum was positively affected by $\mathrm{N}$ intake (Tables 5 and 6), but not by RDP level or energy content of the diet, if the latter is corrected for differences in $\mathrm{N}$ intake. For RDP, this result is not due to inadequate differences between the 60 and $70 \%$ RDP diets, since rumen $\mathrm{NH}_{3}-\mathrm{N}$ concentrations differed highly significantly (Tables 5 and 6).

Meissner \& Du Plessis (1992) reported that NAN passage was greater with the lower $(62 \%)$ RDP diet, but the difference was only significant when compared with the high RDP diet where $\mathrm{N}$ intake was lower and not where $\mathrm{N}$ intake was similar. In the study of Streeter \& Mathis (1995) increased fish meal supplementation increased NAN passage to the duodenum, but the response was due overwhelmingly to an increased $\mathrm{N}$ intake rather than an increased UDP. From a practical point of view, most feedlot diets will be formulated to contain RDP levels between 60 and $70 \%$. Levels of 60 to $65 \%$ RDP are, in fact, considered optimal for feedlot steers (NRC, 1985; Meissner et al., 1992). The quoted and present results suggest that, contrary to belief, NAN passage to the duodenum will not necessarily be higher with $60 \%$ RDP diets than with $70 \%$ RDP diets.

Passage of NAN to the duodenum appears to be, as with starch passage, influenced by the proportion of OM digested in the rumen $\left(O M D_{R}\right)$, which partially is a function of rumen retention time. Two prediction equations where the stepdown procedure was used to eliminate factors that do not contribute significantly to the variation in NAN passage, revealed protein content of the diet or N intake and OMDR as the only major contributing factors. RDP level was rejected. The two prediction equations were:

$$
\begin{aligned}
& \text { (1) NAN }(\mathrm{g} / \text { day })=201.4+0.301 \mathrm{CP}(\%)-1.39 \mathrm{OMD}_{\mathrm{R}}(\%) \\
& r^{2}=0.70 ; S D=16.8 \mathrm{~g} / \text { day }
\end{aligned}
$$

(2) NAN $(g /$ day $)=137.8+0.471 \mathrm{~N}$ intake $(\mathrm{g} /$ day $)-0.013 \mathrm{OMD}_{\mathrm{R}}(\%)$ $r^{2}=0.65 ; S D=17.0 \mathrm{~g} / \mathrm{day}$

The equations indicate that in the present study more NAN passed to the duodenum when proportionally less OM was digested in the rumen. Similar results were obtained with starch passage as discussed. The explanation may be that with less fermentation of OM or starch per unit time more protein will pass undegraded to the duodenum. The animal therefore will benefit twofold if proportionally less $\mathrm{OM}$ is fermented in the rumen. The present data set was limiting in further pursuing the implications of these relationships. Therefore, other data were added to increase the range in protein, RDP and starch contents, in $\mathrm{OMD}_{\mathrm{R}}$ and in NAN and starch passage to the duodenum. The second paper of this series addressed the question again, using the extended data set.

The results of the present investigation support the hypothesis that several combinations of dietary protein, RDP and starch will result in similar quantities of amino acids and glucose in the small intestine, and therefore an optimum dietary protein-energy ratio is unlikely. Additional information is, however, required.

\section{References}

BEEVER, D.E., LOSADA, H.R., GALE, D.L., SPOONER, M.C. \& DHANOA, M.S., 1987. The use of monensin or formaldehyde to control the digestion of the nitrogenous constituents of perennial ryegrass (Lolium perenne cv Melle) and white clover (Trifolium repens $\mathrm{cv}$ Blanca) in the rumen of cattle. Br. J. Nutr. 57, 57.

COLEMAN, S.W., EVANS, B.C. \& HORN, G.W., 1984. Some factors influencing estimates of digesta turnover rate using markers. J. Anim. Sci. 58, 979

CZERKAWSKI, J.W., 1986. An introduction to rumen studies. Pergamon Press, Oxford.

FAICHNEY, G.J., 1980. Measurement in sheep of the quantity and composition of rumen digesta and of the fractional outflow rates and digesta constituents. Aust. J. Agric. Res. 31, 1129.

HENNING, P.H., STEYN, D.G. \& MEISSNER, H.H., 1993. Effect of synchronization of energy and nitrogen supply on ruminal characteristics and microbial growth. J. Anim. Sci. 71, 2516.

HERRERA - SALDANA, R., GOMEZ-ALARCON, R., TORABI, M. \& HUBER, J.T., 1990. Influence of synchronizing protein and starch degradation in the rumen on nutrient utilization and microbial protein synthesis. J. Dairy Sci. 73, 142.

KEARL, L.C., 1982. Nutrient requirements of ruminants in developing countries. Utah State University, Logan. p 120.

KEMPTON, T.J., HILL, M.K. \& LENG, R.A., 1978. The effects of varying bypass amino acids and glucose availability on lamb growth and wool growth. Proc. Austr. Soc. Anim. Prod. 12, 143.

MACRAE, J.C. \& ARMSTRONG, D.G.. 1968. Enzyme method for determination of alpha-linked glucose polymers in biological materials. J. Sci. Fd. Agric. 17, 578.

MACRAE, J.C. \& LOBLEY, G.E., 1986. Interactions between energy and protein. In: Control of digestion and metabolism in ruminants. Eds. Milligan, L.P., Grovum. W.L. \& Dobson, A. Prentice-Hall, New Jersey. p 367.

MEISSNER, H.H. \& DU PLESSIS, P.C., 1992. Protein and starch digestion in steers fed feedlot diets differing in extent of protein degradation. S. Afr. J. Anim. Sci. 22, 137.

MEISSNER, H.H., DU PREEZ, H.P.F. \& DU PLESSIS, P.C., 1992. Effect of level and degradation of dietary protein on performance of feedlot steers. S. Afr. J. Anim. Sci. 22, 128.

MUNTIFERING, R.B., THEURER, B. \& NOON, T.H., 1981. Effects of monensin on site and extent of whole corn digestion and bacterial protein synthesis in beef steers. J. Anim. Sci. 53, 1565.

NEWBOLD, J.R. \& RUST, S.R.. 1990. Effects of protein degradability and source on rumen function, food intake and growth in Holstein cattle given high-moisture maize grain. Anim. Prod. 50, 399.

NRC, 1985. Ruminant nitrogen usage. US National Academy of Science, Washington DC.

OWENS, F.N. \& BERGEN, W.G., 1983. Nitrogen metabolism of ruminant animals: Historical perspective, current understanding 
and future implications. J. Anim. Sci. 57 (Suppl. 2), 498

OWENS, F.N., WEAKLEY, D.C. \& GOETSCH, A.L., 1984.

Modification of rumen fermentation and digestion in the rumen.

In: Herbivore nutrition in the subtropics and tropics. Eds.

Gilchrist, F.M.C. \& Mackie, R.I. The Science Press (Pty) Ltd,

Craighall, South Africa. p 435.

OWENS, F.N., ZINN, R.A. \& KIM, Y.K., 1986. Limits to starch digestion in the ruminant small intestine. J. Anim. Sci. 63, 1634.

PIENAAR, J.P., ROUX, C.Z. \& VAN ZYL, A.B., 1983. A comparison of methods used to estimate a rate constant for outflow from the rumen. S. Afr. J. Anim. Sci. 13, 136.

SAS, 1985. SAS User's Guide, Institute Inc. Raleigh, North Carolina.

SPICER, L.A., THEURER, C.B., SOWE, J. \& NOON, T.H., 1986. Ruminal and postruminal utilization of nitrogen and starch from sorghum grain-, corn- and barley-based diets by beef steers. $J$.
Anim. Sci. 62, 521.

STERN, M.D. \& HOOVER, W.H., 1979. Methods for determining and factors affecting rumen microbial protein synthesis: a review. J. Anim. Sci. 49, 1590.

STORM, E., ØRSKOV, E.R. \& SMART, R.A., 1983. The nutritive value of rumen micro-organisms in ruminants. 2 . The apparent digestibility and net utilization of microbial $\mathrm{N}$ for growing lambs. Br. J. Nutr. 50, 471 .

STREETER, M.N., WAGNER, D.G., OWENS, F.N. \& HIBBERD, C.A., 1991. The effect of pure and partial yellow endosperm sorghum grain hybrids on site and extent of digestion in beef steers. J. Anim. Sci. 69, 2571.

STREETER, M.N. \& MATHIS, M.J., 1995. Effect of supplemented fish meal protein on site and extent of digestion in beef steers. $J$. Anim. Sci. 73, 1196. 